\title{
Effects of 5-HT on Memory and the Hippocampus: Model and Data
}

\author{
Martijn Meeter*, ${ }^{*}$ Lucia Talamini ${ }^{2}$, Jeroen AJ Schmitt ${ }^{3,4}$ and Wim J Riedel ${ }^{3}$ \\ 'Department of Cognitive Psychology, Vrije Universiteit Amsterdam, Amsterdam, The Netherlands; ${ }^{2}$ Department of Psychonomics, Universiteit \\ van Amsterdam, Amsterdam, The Netherlands; ${ }^{3}$ Brain \& Behaviour Institute and Department of Neurocognition, Universiteit Maastricht, \\ Maastricht, The Netherlands; ${ }^{4}$ Nutrition \& Health Department, Nestlé Research Center, Lausanne, Switzerland
}

\begin{abstract}
5-Hydroxytryptamine (5-HT) transmission has been implicated in memory and in depression. Both 5-HT depletion and specific 5-HT agonists lower memory performance, while depression is also associated with memory deficits. The precise neuropharmacology and neural mechanisms underlying these effects are unknown. We used neural network simulations to elucidate the neuropharmacology and network mechanisms underlying 5-HT effects on memory. The model predicts that these effects are largely dependent on transmission over the 5-HTIA and 5- $\mathrm{HT}_{3}$ receptors, which regulate the selectivity of retrieval. It also predicts differential memory deficit profiles for 5-HT depletion and overactivation. The latter predictions were confirmed in studies with healthy and depressed participants undergoing acute tryptophan depletion or ipsipirone challenge. The results suggest that the memory impairments in depressed subjects may be related to 5-HT undertransmission, and support the notion that 5- $\mathrm{HT}_{\text {IA }}$ agonists ameliorate memory deficits in depression.

Neuropsychopharmacology (2006) 31, 7I2-720. doi:I 0.1038/sj.npp. I 300869; published online 3I August 2005
\end{abstract}

Keywords: serotonin, neuromodulation, memory, hippocampus, depression, tryptophan

\section{INTRODUCTION}

Hypofunction of the 5-hydroxytryptamine (5-HT) system has emerged as a leading candidate cause for depression (Naughton et al, 2000; Middlemiss et al, 2002). At the same time, several lines of evidence point to a role for $5-\mathrm{HT}$ in memory (Buhot et al, 2000). Most prominently, depletion of tryptophan (TRP), a precursor of 5-HT, is associated with lower performance on episodic memory retention tests in humans (Riedel et al, 1999). This mirrors findings in depression, which is accompanied by moderate to severe memory deficits (Johnson and Magaro, 1987; Schaub et al, 2003). However, manipulations that increase 5-HT concentration or 5-HT receptor activation also lower memory performance, as indicated by receptor agonist studies in both humans (Riedel et al, 2002) and animals (Altman and Normile, 1988). Up to now, it is not known why both increased and decreased 5-HT activation have adverse effects on memory.

5-HT binds to a large and disparate family of CNS receptors, which have effects that are sometimes opposite.

\footnotetext{
*Correspondence: Dr M Meeter, Department of Cognitive Psychology, Vrije Universiteit Amsterdam, Vd Boechorststraat I, I08I BT Amsterdam, The Netherlands, Tel: + 3120 4282906,

E-mail: m.meter@uva.nl

Received I 8 March 2005; revised I June 2005; accepted 27 June 2005 Online publication: 14 July 2005 at http://www.acnp.org/citations/ Npp07| 405050192/default.pdf
}

Thus, many effects of different receptors seem to cancel each other out. In a review (see Supplementary Information on internet), we identify two robust effects of 5-HT in the hippocampus. First, 5-HT exerts a hyperpolarizing influence on principal cells; directly, via $5-\mathrm{HT}_{1 \mathrm{~A}}$ receptors, and indirectly, via facilitation of GABA release from local interneurons through 5- $\mathrm{HT}_{3}$ receptors (Burnet et al, 1995; Piguet and Galvan, 1994). Activation of 5- $\mathrm{HT}_{2 \mathrm{~A}}$ and $5-\mathrm{HT}_{2 \mathrm{C}}$ receptors has been suggested to induce depolarization in principal cells (Piguet and Galvan, 1994; Barnes and Sharp, 1999), but these effects appear to be dominated by the depolarizing effects of 5-HT, as bath application of 5-HT will hyperpolarize principal cells in slice preparations of the dentate gyrus (Piguet and Galvan, 1994). In addition, through $5-\mathrm{HT}_{2 \mathrm{C}}, 5-\mathrm{HT}_{4}$, and $5-\mathrm{HT}_{7}$ receptors, afterhyperpolarizing (AHP) currents are downregulated, leading to reduced adaptation in principal cells (Torres et al, 1996; Bacon and Beck, 2000). The increased firing rate observed in slices after prolonged application of 5-HT has been linked to this mechanism (Andrade and Nicoll, 1987; Andrade and Chaput, 1991).

To investigate the actions of 5-HT on memory, we turned to computational modelling of the hippocampus, a medial temporal lobe structure known to underlie episodic memory. There are several extant neurocomputational models of this structure, which agree to a substantial level on the essentials of hippocampal involvement in episodic memory (Gluck et al, 2003). One such model (see Figure 1) 


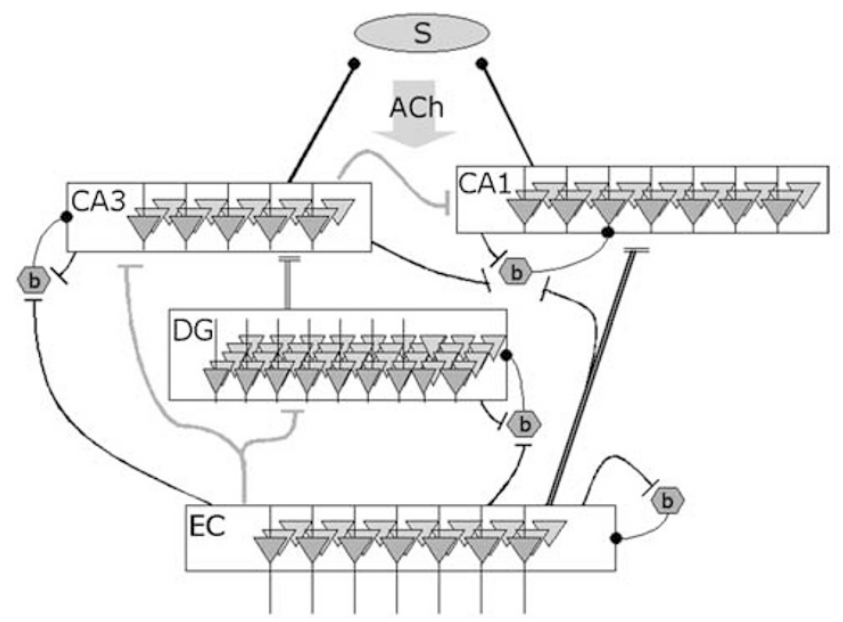

Figure I Hippocampal model used in the simulations. Four layers model the entorhinal cortex (EC), dentate gyrus (DG), and fields CAI and CA3 of the hippocampus proper. The model contains 80 EC nodes, 320 DG nodes, 68 CA3 nodes, and 100 CAI nodes, with in addition one basket cell (marked with b) per layer. The basket cells receive input from both the layer they reside in (feedback inhibition) and layers that project to it (feedforward inhibition). Flat connection endings are excitatory, while round ones are inhibitory. Solid connections are fanning, while open ones are point-to-point, with each sender targeting only one (EC to CAI) or a few (DG to CA3) receiving nodes. The septum, controlled by inhibition from the hippocampus, releases ACh to all nodes in the system.

has recently been applied to study the effects of acetylcholine on the hippocampus and its role in memory (Meeter et al, 2004). We adapted it to investigate the effects of 5-HT on that same structure, by incorporating both effects of 5-HT discussed above. We then simulated a typical episodic memory task at different levels of 5-HT, ranging from depletion to overactivation. The single free parameter in these simulations was the relative strength of the two net effects of 5-HT, hyperpolarization and reduced adaptation, on which there is no biological data. The contribution of each effect to hippocampal processing was investigated by varying its strength independently. The predictions of the simulations were subsequently tested with data from two psychopharmacological studies: one in which the effect of TRP depletion was investigated, and one in which patients were administered $5-\mathrm{HT}_{1 \mathrm{~A}}$ agonists.

\section{MATERIALS AND METHODS}

\section{Model}

Except for the addition of simulated 5-HT receptors, no changes were made to a published model of the hippocampus (Meeter et al, 2004). The model was built using spiking McGregor model neurons. These integrate-and-fire nodes are abstracted from the Hodgkin-Huxley formalism and incorporate sodium, potassium, and chloride currents, implementing excitatory and inhibitory inputs, leak currents, and adaptation (MacGregor and Oliver, 1974). They were simulated in discrete time steps of $2 \mathrm{~ms}$. Hebbian learning was used, with the addition of negative Hebbian learning that models LTD. Model architecture and parameter values were based, where possible, on biological data.

The model consisted of four layers representing subfields of the hippocampal formation (Figure 1). An entorhinal cortex layer (EC) functioned as the input layer of the model. The next layer represented the granule layer of the dentate gyrus (DG). Of the hippocampus proper, fields CA3 and CA1 were included. CA1 functioned as the output module of the model. Per layer, one inhibitory node was implemented to sustain feedforward and feedback inhibition. The connectivity between the model layers reflects the known pathways in the hippocampus (for a review, see Witter et al, 2000).

Two kinds of oscillations were incorporated in the model, fast $\gamma$ band oscillations $(20-60 \mathrm{~Hz})$ and slower $\theta$ band oscillations $(4-12 \mathrm{~Hz}) . \theta$ wave oscillations in the model resulted from inhibitory oscillators, known to exist in the medial septum, which project to hippocampal inhibitory cells (Acsády et al, 1993; Freund and Antal, 1988; Gulyás et al, 1991; Gulyás et al, 1990). Rhythmic firing of these oscillators phasically disinhibits hippocampal principal cells at $\theta$ frequency (Tóth et al, 1997). $\gamma$ frequency oscillations arose within the modeled layers from the interplay of excitation and fast feedback inhibition.

Also modelled was a medial septal cholinergic node. This node controlled the release of acetylcholine (ACh) in all modelled layers. The node itself received input from the septal $\theta$ oscillator, and from hippocampal interneurons (Dragoi et al, 1999; McLennan and Miller, 1974; Tóth et al, 1993). The latter connections were inhibitory; in essence, the cholinergic node was disinhibited only when entorhinal input did not elicit any recall in the hippocampus; that is, when the input was new. This allowed the model to automatically switch between two modes, a learning mode characterized by a high $\mathrm{ACh}$ release, and a retrieval mode characterized by a low ACh release. As ACh stimulates the formation of new patterns and also enhances LTP (Hasselmo, 1995; Hasselmo and Bower, 1993), such dynamics favor the formation of new representations in case of new input, and the maintenance of old representations in case of old input (Meeter et al, 2004).

\section{Simulations}

We repeated the basic simulation of pattern learning and retrieval reported earlier (Meeter et al, 2004). An entorhinal input pattern $A$, consisting of 12 nodes, is activated during one $\theta$ cycle in learning mode, characterized by a high ACh level. This leads to new hippocampus representations being formed through competitive learning in the dentate and CA3 layers. Converging CA3 and EC inputs activate CA1 nodes, which store the correspondence of the EC input pattern and its CA3 representation.

After the acquisition phase, both the stored pattern $A$, and a new input pattern $B$ are presented to the model during one $\theta$ oscillation in retrieval mode, characterized by a low ACh level. Presentation of pattern $B$ is included to guarantee the specificity of retrieval (the activity it elicited increased and decreased in line with incorrect retrieval, and will not be separately reported). As the connections between EC and CA1 are one-to-one, only 12 CA1 nodes are innervated by the EC input pattern. The maximum number of these 12 CA1 nodes that are simultaneously active during retrieval constitutes the measure of correct recall. The maximum number of other CA1 nodes that are simultaneously active during retrieval constituted our 'incorrect nodes' measure. 
The level of 5-HT was assumed to remain constant during the simulation, and was represented by a variable $\phi$, with a value of 1 representing the normal situation. The hyperpolarizing effects of 5-HT on hippocampal principal nodes were instantiated by an inhibitory current $-\alpha \phi$ added to the input to principal cells, which stands for the 5-HT level multiplied by a constant $\alpha$. The attenuation of AHP was implemented through modification of the adaptation constant $\tau: \tau=\tau_{\mathrm{s}} / \phi$, with $\tau_{\mathrm{s}}$ representing the standard value of the time constant. For other model details, see the appendix.

\section{Depletion study}

TRP is the precursor of 5-HT. Acute TRP depletion causes a dramatic lowering of both plasma concentrations of free TRP and the rate of 5-HT synthesis in the human brain. These effects occur rapidly, within 4 and $6 \mathrm{~h}$ for TRP and 5-HT, respectively, due to the rate-limiting enzyme TRP hydroxylase, which converts TRP to 5-HT (Riedel, 2004).

A total of 17 healthy volunteers drank $100 \mathrm{~g}$ of an aminoacid mixture with or without TRP, according to a doubleblind, crossover design. We report memory performance $5 \mathrm{~h}$ after administration. Tests at longer delays following administration are not reported, but show the same pattern. More details are reported elsewhere (Schmitt et al, 2000).

\section{Agonist study}

A total of 15 patients with major depression, dysthymia, or anxiety disorder with depressed mood (DSM-IV diagnoses) and 16 controls received on three consecutive days single oral doses of $10 \mathrm{mg}$ ipsapirone, a $5-\mathrm{HT}_{1 \mathrm{~A}}$ agonist, or $0.5 \mathrm{mg} / \mathrm{kg}$ metachlorophenylpiperazine (m-CPP), a $5-\mathrm{HT}_{2 \mathrm{C}}$ agonist, according to a double-blind, placebo-controlled, crossover design. Participants then completed a memory task, yielding immediate and delayed recall and recognition scores. The metachlorophenylpiperazine condition is not reported here, as our model does not predict strong memory deficits from stimulation of $5-\mathrm{HT}_{2 \mathrm{C}}$ receptors. Indeed, no substantial effects of $\mathrm{m}$-CPP were found. More details are reported elsewhere (Riedel et al, 2002).

\section{RESULTS}

In the simulations, the model stores a pattern in a learning phase, and later, in a retrieval phase, retrieves it. Figure 2 shows a normalized summation of activity in CA1 and CA3 during the retrieval phase, at different levels of simulated 5-HT action. Increased hyperpolarization through 5-HT causes a drop in pyramidal firing. Simultaneously, the decreased adaptation that also characterizes 5-HT's effects in the hippocampus leads to higher firing rates, as has been found in vitro (Andrade and Nicoll, 1987; Andrade and Chaput, 1991).

The model can make two kinds of errors, errors of omission and errors of commission. In the first case, it does not retrieve part of the stored pattern, in the second it activates, during retrieval, nodes that do not belong to the pattern. Figure 3 shows how hippocampal retrieval of information is affected by simulated changes in 5-HT action, for different relative strengths of the two effects of

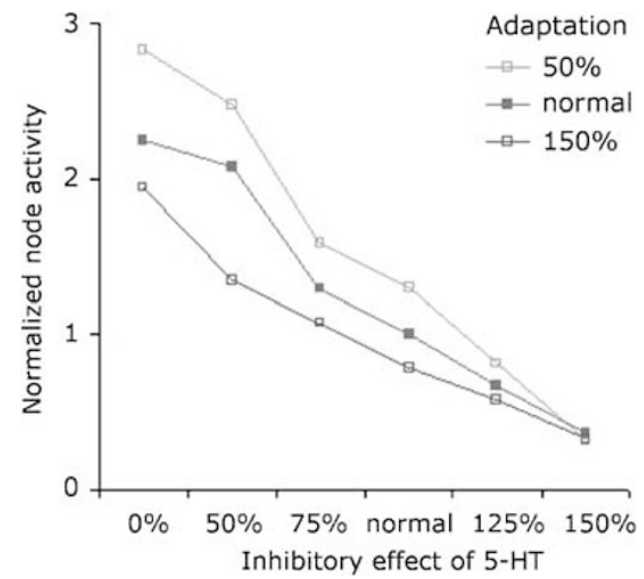

Figure 2 Normalized activity level in layers CAI and CA3 of the model, during one $\theta$ cycle of retrieval at different levels of simulated 5-HT action. $X$-axis gives the level of principal cell hyperpolarization exerted by $5-\mathrm{HT}$, different lines represent different levels of adaptation. Activity is normalized by dividing simulated spike counts by the number of simulated spikes at the normal 5-HT level.

5-HT. Panel a shows activation of correct nodes during retrieval, panel $b$ the activation of incorrect nodes (those not belonging to the stored pattern), while panel $\mathrm{c}$ shows a composite recall index, which encompasses both aforementioned measures.

Changes in adaptation have relatively little effect on performance, as evidenced by the virtually equal results for the three levels of adaptation. In contrast, hyperpolarization of principal cells through 5-HT does affect memory performance. Strong hyperpolarization leads to a drop in correct retrieval. Conversely, a low level of hyperpolarization spares retrieval of correct nodes, but facilitates activation of incorrect nodes (panel $b$ ) to the extent that the integrity of retrieval suffers. Both higher and lower than normal levels of hyperpolarization thus lead to a decrease in our retrieval index (panel c), leading to an inverted U-curve of memory performance as a function of 5-HT action. This reproduces the finding, discussed earlier, that 5-HT depletion as well as excessive stimulation of 5-HT receptors hinders episodic recall. Our results suggest that both effects depend largely on hyperpolarization through $5-\mathrm{HT}_{1 \mathrm{~A}}$ and $5-\mathrm{HT}_{3}$ receptors, and not on changes in AHP currents. Stimulation of $5-\mathrm{HT}_{1 \mathrm{~A}}$ and $5-\mathrm{HT}_{3}$ receptors mostly affects the quantity of information retrieved, and 5-HT depletion mostly its quality.

Successful episodic recall depends on both the quantity and the quality of information retrieved from memory. However, recognition may rely predominantly on the quantity of information retrieved (Humphreys et al, 1989), with recognition hit rates roughly indexing correctly retrieved information and false alarm rates the amount of incorrectly retrieved information. Our modelling work thus predicts that lowered 5-HT levels will lead to recall deficits, normal hit rates (as the quantity of correctly retrieved nodes should not be affected), and increased false alarm rates. On the other hand, stimulation of 5-HT receptors should lead to recall deficits, lower hit rates, and lower false alarm rates. The modelling thus predicts that each condition is accompanied by a distinct memory profile. 

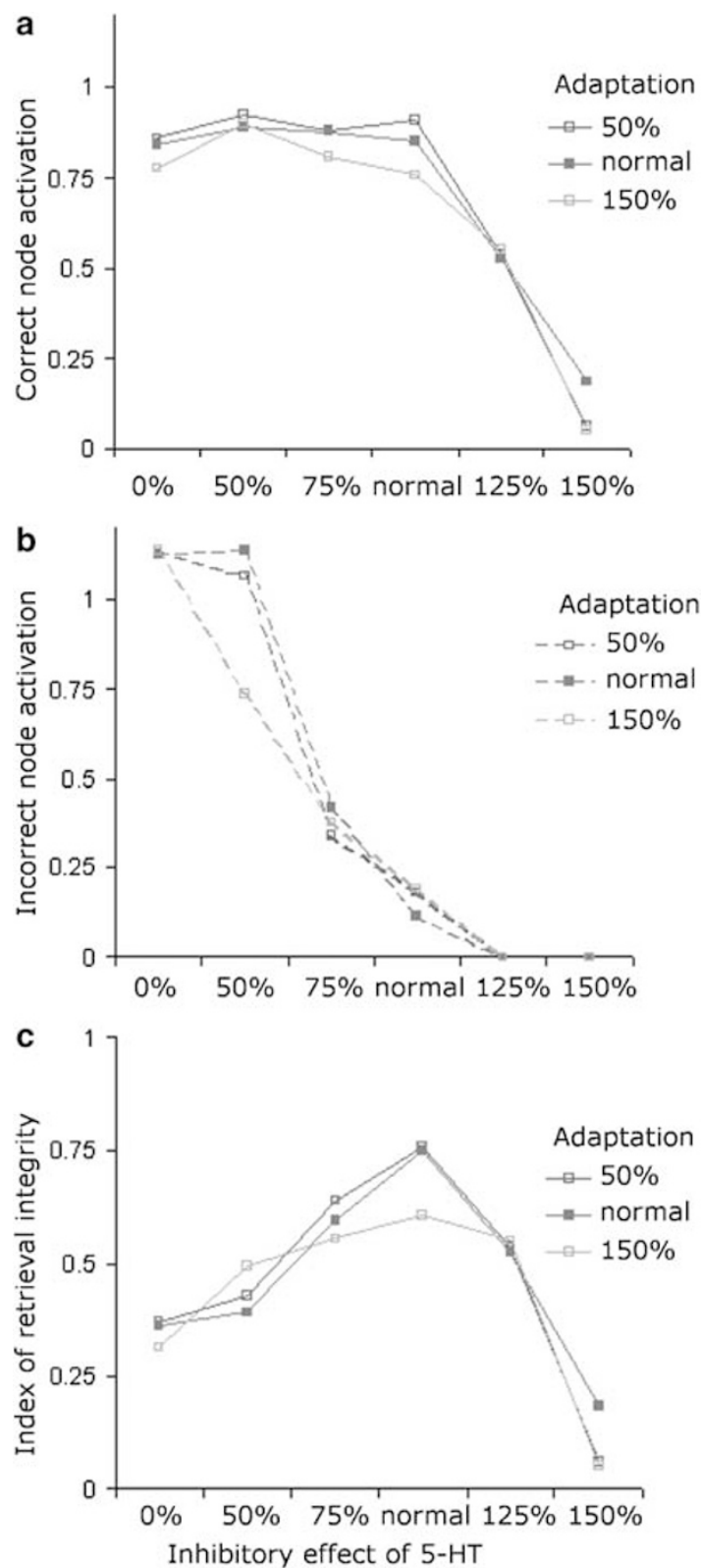

Figure 3 Memory performance of the model. (a) Maximum number of simultaneously active pattern nodes in CAI during one $\theta$ cycle of retrieval at different levels of simulated 5-HT action. X-axis gives the level of principal cell hyperpolarization exerted by $5-\mathrm{HT}$, different lines represent different levels of adaptation. (b) Maximum number of incorrect nodes activated during one $\theta$ cycle of retrieval. (c) Index of memory retrieval, calculated as $C *(C /(C+E))$, where $C$ is the number of correctly retrieved nodes shown in panel $a$, and $E$ is the number of incorrectly activated nodes shown in panel $b$.

\section{Experiments}

To test these predictions, we reanalyzed data of experiments in which standardized memory batteries, including immediate and delayed recall and recognition tests, were administered in either 5-HT depletion or 5-HT stimulation conditions. While previous reports on these and similar experiments considered recall and measures of recognition sensitivity, we now determined the separate effects of each condition on hit rates and false alarm rates.

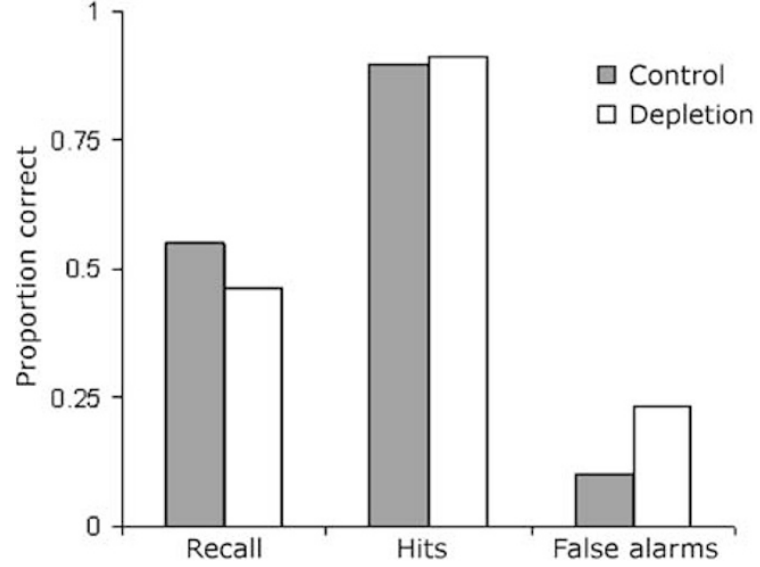

Figure 4 Performance on the Visual Verbal Learning Test, in which I5 words first have to be recalled in a free recall format, and are then tested in a recognition format. Presented are the results from 17 normal controls, tested under acute TRP depletion conditions and under a control condition in which TRP intake was normal.

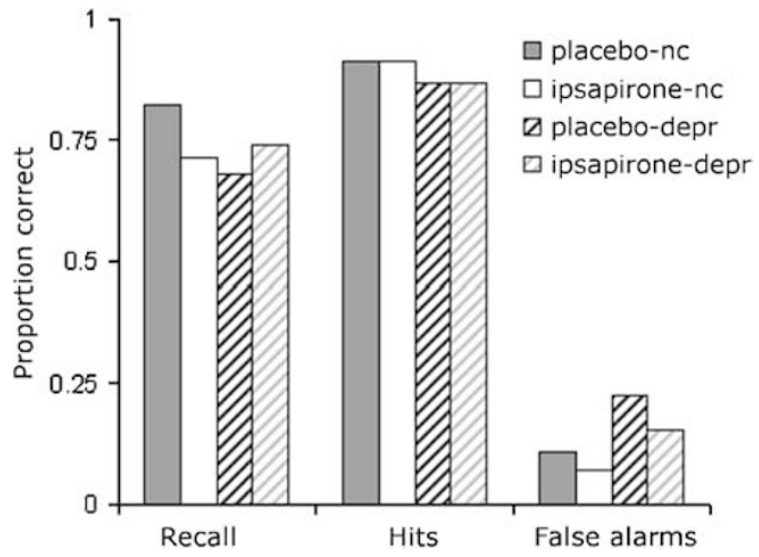

Figure 5 Performance on a word-learning test in which 15 words were presented for five trials, and subsequently tested in recall and recognition format. Presented are the results from 15 patients with unipolar depression (shaded bars marked 'depr.') and 16 normal controls (solid bars marked ' $n c$ '), tested after oral ingestion of either ipsapirone or a placebo (control).

In the depletion study, healthy participants were tested, on different days, following TRP depletion and in a control condition. It has already been shown that healthy volunteers' delayed recall and recognition of newly learnt material were specifically impaired after acute TRP depletion (Schmitt et al, 2000). We looked at results in more detail, analyzing hit rates and false alarm rates separately (Figure 4). As predicted, recognition hit rates were not lowered in the depletion condition, $t(15)=0.565, p=0.580$, and false alarm rates were higher in the depletion condition than in the control condition, $t(15)=2.988, p=0.009$, two-tailed.

For the 5-HT agonist study, we analyzed recall scores, as well as hit rates and false alarm rates in recognition (Figure 5). On immediate recall, there were no main effects of condition, $\mathrm{F}<1$, or group, $\mathrm{F}(1,27)=1.45, p=0.24$, but there was a crossover interaction between these two factors, $\mathrm{F}(1,29)=6.85, p<0.014$. Whereas normal controls performed worse with ipsapirone, performance of depressed participants improved. In delayed recall, neither main 
effects nor the interaction were significant, but the pattern in means was in the same direction as for immediate recall. These results are in line with predictions from the simulations, given the common view of depression as involving 5- $\mathrm{HT}_{1 \mathrm{~A}}$ hypoactivity (Naughton et al, 2000). Ipsapirone shifts persons with depression from the low side of the inverted U-curve in Figure $3 c$ to the right and upward, leading to better recall. Normal controls are shifted from the top of the curve to the right and downward, leading to decreased recall.

On hit rates, there were no main effects of either group, $\mathrm{F}(1,27)=2.565, p=0.12$, or condition, $\mathrm{F}<1$, nor was there an interaction between the two. False alarm rates were lower for control participants than for depressed participants, $\mathrm{F}(1,27)=5.90, p=0.022$. In both groups, they were lower in the ipsapirone condition than in the control condition, $\mathrm{F}(1,27)=5.01, p=0.034$ (there was no interaction between group and condition). Again, this is in line with predictions from the simulations, first in that persons with depression, more to the left in Figure $3 \mathrm{~b}$, have increased levels of false recognition, and second, in that increasing $5-\mathrm{HT}_{1 \mathrm{~A}}$ activation (a shift to the right in Figure $3 \mathrm{~b}$ ) lowers retrieval of false information in both groups.

\section{DISCUSSION}

According to our simulations, 5-HT influences memory performance through its hyperpolarizing effects, whereas changes in adaptation may not have a large influence on memory performance. Distinct memory profiles were derived for low and high transmission of 5-HT in the hippocampus: low 5-HT transmission was predicted to lead to a relatively large amount of information being retrieved, at the cost of activating irrelevant information and of high levels of false alarms. High 5-HT transmission was predicted to lead to a reduced quantity of retrieved information, leading to lower recall scores combined with lower levels of false alarms. These predictions were verified with data from psychopharmacological experiments. Lowered levels of 5-HT transmission were operationalized as TRP depletion, increased transmission as an ipsapirone challenge. (Ipsapirone will not only work through postsynaptic $5-\mathrm{HT}_{1 \mathrm{~A}}$ receptors but may also, via autoreceptors in the raphe nucleus, lower 5-HT release (Dong et al, 1997). Even if the latter effect is strong, there may well be a net increase in hyperpolarization, as a lower 5-HT release decreases both hyperpolariziation through $5-\mathrm{HT}_{1 \mathrm{~A}}$ and $5-\mathrm{HT}_{3}$ receptors, and depolarization through $5-\mathrm{HT}_{2 \mathrm{~A}}$ and $5-\mathrm{HT}_{2 \mathrm{C}}$ receptors, whereas postsynaptic effects of ipsapirone are specific to $5-\mathrm{HT}_{1 \mathrm{~A}}$.) As predicted, we found increased false alarm rates during TRP depletion, and lowered false alarm rates after administration of ipsapirone. A further result, whereby ipsapirone increased recall in depressed participants but lowered it in normal controls, was also in line with predictions from the simulations.

Our study is unusual in taking hit and false alarm rates as basic recognition memory measures. Most often, results from recognition tests are reported using sensitivity and bias measures, such as $d^{\prime}$ 'and $\beta$ (Banks, 1970; Snodgrass and Corwin, 1988). These measures are computed from hit and false alarm rates, and changes in false alarm and hit rates can also be reported as decreases and increases in sensitivity and bias. We feel that for our results this would not increase clarity, however. Findings in recognition memory usually fit various 'mirror effects' (Glanzer and Adams, 1985; Glanzer et al, 1993; Ruiz et al, 2004): while hit rates go down false alarm rates go up, and vice versa. Our data do not conform to this rule: as false alarm rates went up or down, hit rates remained stable. This can only be explained by peculiar combinations of sensitivity and bias changes, which suggests that a change in false alarm rates is not secondary to an underlying change in sensitivity only.

In the simulations, the effects of 5-HT were varied during both the acquisition and the testing phase. This was done because the same conditions were applied in the experiments. We performed additional simulations in which 5-HT was held constant during either acquisition or retrieval, and varied during the other phase. For both the 5-HT depletion and stimulation conditions, effects were strongest when 5 -HT was increased during the acquisition phase. Nevertheless, in both conditions, smaller effects in the same direction were also found when 5-HT was manipulated only during retrieval. For 5-HT depletion, there are indications that effects are indeed stronger when the depletion occurs before learning, rather than following it (Schmitt et al, 2000). The prediction that the larger effect of 5-HT stimulation occur at acquisition remains to be tested.

Several complications of 5-HT neuropharmacology have not been incorporated in this study. One is the influence that the 5-HT system exerts on cortisol release. Pharmacological manipulations of 5-HT, including ipsapirone challenge, are known to induce a cortisol response through the hypothalamic-pituitary-adrenal (HPA) axis. This is especially pertinent, given suggestions that depressed individuals show a blunted cortisol response to ipsapirone challenge (Meltzer and Maes, 1995; Riedel et al, 2002). Whether this cortisol blunting results in differential effects on episodic memory is not clear. Cortisol is sometimes reported to enhance learning of neutral material, but also to impair it (Abercrombie et al, 2003; Jelicic et al, 2004). This could imply that memory impairment seen in healthy volunteers after ipsapirone, but also after all other serotonergic challenge agents, might theoretically be ascribed to elevation of cortisol. However, Mcpp induced a similar cortisol response as ipsapirone, but did not affect memory in either patients or controls. Furthermore, since the cortisol response to ipsapirone was slightly blunted in depressed patients, this could at most explain a decrease in memory impairment relative to controls. In fact, immediate recall performance was improved after ipsapirone in depressed patients (Riedel et al, 2002).

Another issue is that ipsapirone may not be wholly specific to $5-\mathrm{HT}_{1 \mathrm{~A}}$ receptors, but may affect the noradrenergic system as well, either through its low affinity for alfa2 receptors or through metabolites such as 1-PP (Yatham and Steiner, 1993; Piercey et al, 1994). However, none of this has ever been substantiated in humans by showing measures of association between correlates of alfa2-binding and behavioural effects or serotonergic-noradrenergic interaction studies. In a study of the interaction of ATD and buspirone (a $5-\mathrm{HT}_{1 \mathrm{~A}}$ agonist with higher reported affinities of alfa2 as well as a resultant 1-PP metabolite), it was shown that 1-PP blood levels were not associated to any of the behavioral 
and physiological effects of buspirone, making it less likely that this metabolite is primarily responsible for the observed effects of $5-\mathrm{HT}_{1 \mathrm{~A}}$ agonists (Jagannathan and Venitz, 1997). In addition, these authors also showed that ATD pretreatment attenuated the behavioral and physiological effects of buspirone, confirming the impression that the azapirone 5- $\mathrm{HT}_{1 \mathrm{~A}}$ agonists like buspirone and ipsapirone act primarily through the 5-HT system. The affinity of ipsapirone for $5-\mathrm{HT}_{1 \mathrm{~A}}$ receptors is quite dominant over other affinities (Peroutka, 1988). Both of these issues touch on the relations between the 5-HT system and other modulatory systems, a larger issue that we hope to pick up in further studies.

The results reported here in first instance apply to studies in which 5-HT and its receptors are manipulated in healthy controls. Interest in these studies is for a large part driven by the connection between 5-HT and depression. This raises the question whether the results also have relevance for the study of depression; in particular, whether simulation findings for low 5-HT can help in understanding memory deficits found in depression. These deficits might just be secondary to mood, but the idea that depression is accompanied by neuropsychological deficits independent of mood is gaining wider currency (Fleming et al, 2004). A link between 5 -HT and memory deficits in depression is suggested by the fact that serotonergic antidepressants have been found to improve memory before mood improved (Allain et al, 1992; Fudge et al, 1990; Siegfried and O'Connolly, 1986). In our discussion of the ipsapirone study, we relied on the hypothesis that hypoactivity of $5-\mathrm{HT}_{1 \mathrm{~A}}$ receptors causes part or all of the memory deficits seen in depression. Based on our results and known 5-HT dysregulation in depression (Naughton et al, 2000), we find this a defendable hypothesis, although more evidence is clearly needed to link memory deficits in depression to 5-HT with any certainty.

It is also tempting to speculate on the effects that antidepressant medication may have on memory through manipulations of 5-HT. Selective serotonergic reuptake inhibitors (SSRIs) will increase, at least acutely, levels of 5 -HT in the central nervous system (Anderson et al, 2005). This could be expected to alter hippocampal processing in ways consistent with a shift to the right in the panels of Figure 3. In particular, free recall should suffer in normal controls but improve in depressed patients, while false alarms should decrease in both groups. Most studies of specific serotonergic antidepressant effects on memory show no acute effect. For some SSRIs, impairment of delayed recall has been found after 2 weeks of treatment with therapeutic doses (Riedel et al, 2005; Schmitt et al, 2001). However, there is one study demonstrating an acute improvement of delayed recall after the intravenous administration of citalopram $10 \mathrm{mg}$ (Harmer et al, 2002). There is one further clinical study showing that after 6 weeks of treatment, the noradrenergic antidepressant desipramine and the SSRI fluoxetine were found to be equally effective in terms of their clinical effects on mood (Levkovitz et al, 2002). Improvement of memory, however, was only apparent with the serotonergic antidepressant fluoxetine. This not only suggests a dissociation between clinical response and memory improvement but is also in keeping with our proposed role of 5-HT in memory processes.
Few hypotheses exist about the function of 5-HT in normal memory. Our simulations suggest that 5-HT may allow criterion shifting during storage and memory search: a low level of 5-HT in the hippocampus may constitute a permissive criterion for storage and retrieval, a higher level of 5-HT in the hippocampus a stricter criterion. In situations of 5-HT depletion, possibly including depression, the hippocampus may continuously be in a state favoring retrieval of irrelevant information, hampering recall performance and increasing false alarm rates. As in our model, effects at the $5-\mathrm{HT}_{1 \mathrm{~A}}$ receptor are important in connecting 5-HT to memory performance, this suggests a theoretical ground for the finding that $5-\mathrm{HT}_{1 \mathrm{~A}}$ agonists can relieve memory deficits in depression (Riedel et al, 2002).

\section{ACKNOWLEDGEMENTS}

The research of M Meeter was supported by grant 402-01630 from the Dutch National Science Foundation (NWO). We thank Arjan Blokland for stimulating discussions. The contribution of $\mathrm{W}$ Riedel and interpretation of research data for this article were entirely carried out in the University of Maastricht. Currently, W Riedel is also affiliated to GlaxoSmithKline R\&D.

\section{REFERENCES}

Abercrombie HC, Kalin NH, Thurow ME, Rosenkranz MA, Davidson RJ (2003). Cortisol variation in humans affects memory for emotionally laden and neutral information. Behav Neurosci 117: 505-516.

Acsády L, Halasy K, Freund TF (1993). Calretinin is present in non-pyramidal cells of the rat hippocampus-III. Theri inputs from the median raphe and medial septal nuclei. Neuroscience 52: 829-841.

Allain H, Lieuniy A, Brunet BF, Mirabaud C, Trebon P, LeCoz F et al (1992). Antidepressants and cognition: comparative effects of moclobemide, viloxazine and maprotiline. Psychopharmacol Suppl 106: 58-61.

Altman HJ, Normile HJ (1988). What is the nature of the role of the serotonergic nervous system in learning and memory: prospects for development of an effective treatment strategy for senile dementia. Neurobiol Aging 9: 627-638.

Anderson GM, Barr CS, Lindell S, Durham AC, Shifrovich I, Higley JD (2005). Time course of the effects of the serotonin-selective reuptake inhibitor sertraline on central and peripheral serotonin neurochemistry in the rhesus monkey. Psychopharmacology (Berlin) 178: 339-346.

Andrade R, Chaput Y (1991). 5-Hydroxytrytamine4-like receptors mediate the slow excitatory response to serotonin in the rat hippocampus. J Pharmacol Exp Ther 257: 930-937.

Andrade R, Nicoll RA (1987). Pharmacologically distinct actions of serotonin in the rat hippocampus. J Physiol 394: 99-124.

Bacon WL, Beck SG (2000). 5-Hydroxytryptamine(7) receptor activation decreases slow afterhyperpolarization amplitude in CA3 hippocampal pyramidal cells. J Pharmacol Exp Ther 294: 672-679.

Banks WP (1970). Signal detection theory and human memory. Psychol Bull 74: 81-99.

Barnes NM, Sharp T (1999). A review of central 5-HT receptors and their function. Neuropharmacology 38: 1083-1152.

Buhot MC, Martin S, Segu L (2000). Role of serotonin in memory impairment. Ann Med 32: 210-221. 
Burnet PWJ, Eastwood SL, Lacey K, Harrison PJ (1995). The distribution of 5-HT1A and 5-HT2A receptor mRNA in human brain. Brain Res 676: 157-168.

Dong J, de Montigny C, Blier P (1997). Effect of acute and repeated versus sustained administration of the 5-HT1A receptor agonist ipsapirone: electrophysiological studies in the rat hippocampus and dorsal raphe. Naunyn-Schmiedeberg Arch Pharmacol 356: 303-311.

Dragoi G, Carpi M, Recce M, Csicsvari J, Buzsaki G (1999). Interactions between hippocampus and medial septum during sharp wave and theta oscillation in the behaving rat. $J$ Neurosci 19: 6191-6199.

Fleming SK, Blasey C, Schatzberg AF (2004). Neuropsychological correlates of psychotic features in major depressive disorders: a review and meta-analysis. J Psychiatr Res 38: 27-35.

Freund T, Antal M (1988). GABA-containing neurons in the septum control inhibitory interneurons in the hippocampus. Nature 336: 170-173.

Fudge JL, Perry PJ, Garvey MJ, Kelly MW (1990). A comparision of the effect of fluoxetine and trazodone on the cognitive functioning of depressed outpatients. J Affective Disorders 18 275-280.

Glanzer M, Adams JK (1985). The mirror effect in recognition memory. Mem Cognit 13: 8-20.

Glanzer M, Adams JK, Iverson GJ, Kim K (1993). The regularities of recognition memory. Psychol Rev 100: 546-567.

Gluck MA, Meeter M, Myers CE (2003). Computational models of the hippocampal region: linking incremental learning and episodic memory. Trends Cogn Sci 7: 269-276.

Gulyás AI, Görcs TJ, Freund TF (1990). Innervation of different peptide-containing neurons in the hippocampus by GABAergic septal afferents. Neuroscience 37: 31-44.

Gulyás AI, Seress L, Toth K, Acsady L, Antal M, Freund TF (1991). Septal GABAergic neurons innervate inhibitory interneurons in the hippocampus of the macaque monkey. Neuroscience 41: 381-390.

Harmer CJ, Bhagwagar Z, Cowen PJ, Goodwin GM (2002). Acute administration of citalopram facilitates memory consolidation in healthy volunteers. Psychopharmacology (Berlin) 163: 106-110.

Hasselmo ME (1995). Neuromodulation and cortical function: modeling the physiological basis of behavior. Behav Brain Res 67: 1-27.

Hasselmo ME, Bower JM (1993). Acetylcholine and memory. Trends Neurosci 16: 218-222.

Hasselmo ME, Fehlau BP (2001). Differences in time course of ACh and GABA modulation of excitatory synaptic potentials in slices of rat hippocampus. J Neurophysiol 86: 1792-1802.

Hasselmo ME, Schnell E, Barkai E (1995). Dynamics of learning and recall at excitatory recurrent synapses and cholinergic modulation in rat hippocampal region CA3. J Neurosci 15: 5249-5262.

Hodgkin AL, Huxley AF (1952). A quantitative description of ion currents and its application to conduction and excitation in nerve membranes. J Physiol 117: 500-544.

Humphreys MS, Bain JD, Pike R (1989). Different ways to cue a coherent system: a theory for episodic, semantic and procedural tasks. Psychol Rev 96: 208-233.

Jagannathan V, Venitz J (1997). Pharmacokinetics and CNS pharmacodynamics of the 5-HT1A agonist buspirone in humans following acute L-tryptophan depletion challenge. Methods Find Exp Clin Pharmacol 19: 351-361.

Jelicic M, Geraerts E, Merckelbach H, Guerrieri R (2004). Acute stress enhances memory for emotional words, but impairs memory for neutral words. Int J Neurosci 114: 1343-1351.

Johnson MH, Magaro PA (1987). Effects of mood and severity on memory processes in depression and mania. Psychol Bull 101: 28-40.

Levkovitz Y, Caftori R, Avital A, Richter-Levin G (2002). The SSRIs drug Fluoxetine, but not the noradrenergic tricyclic drug
Desipramine, improves memory performance during acute major depression. Brain Res Bull 15: 345-350.

MacGregor RJ, Oliver RM (1974). A model for repetitive firing in neurons. Cybernetik 16: 53-64.

McLennan H, Miller JJ (1974). The hippocampal control of neuronal discharges in the septum of the rat. $J$ Physiol 237: 607-624.

Meeter M, Talamini LM, Murre JMJ (2004). Mode shifting between storage and recall based on novelty detection in oscillating hippocampal circuits. Hippocampus 14: 722-741.

Meltzer HY, Maes M (1995). Effects of ipsapirone on plasma cortisol and body temperature in major depression. Biol Psychiatry 38: 450-457.

Middlemiss DN, Price GW, Watson JM (2002). Serotonergic targets in depression. Curr Opin Pharmacol 2: 18-22.

Naughton M, Mulrooney JB, Leonard BE (2000). A review of the role of serotonin receptors in psyciatric disorders. Hum Psychopharmacol 15: 397-415.

Peroutka SJ (1988). 5-Hydroxytryptamine receptor subtypes: molecular, biochemical and physiological characterization. Trends Neurosci 11: 496-500.

Piercey MF, Smith MW, Lum-Ragan JT (1994). Excitation of noradrenergic cell firing by 5-hydroxytryptamine1A agonists correlates with dopamine antagonist properties. J Pharmacol Exp Ther 268: 1297-1303.

Piguet P, Galvan M (1994). Transient and long-lasting actions of 5-HT on rat dentate gyrus neurones in vitro. J Physiol 481: 629-639.

Riedel WJ (2004). Editorial: Cognitive changes after acute tryptophan depletion; what can they tell us? Psychol Med 34: 3-8.

Riedel WJ, Eikmans K, Heldens A, Schmitt JAJ (2005). Specific serotonergic reuptake inhibition impairs vigilance performance acutely and after subchronic treatment. J Psychopharmacol 19: $12-20$.

Riedel WJ, Klaassen T, Deutz NEP, van Someren A, van Praag HM (1999). Tryptophan depletion in normal volunteers produces selective impairment in memory consolidation. Psychopharmacology 141: 362-369.

Riedel WJ, Klaassen T, Griez E, Honig A, Menheere PPCA, van Praag HM (2002). Dissociable hormonal, cognitive and mood responses to neuroendocrine challenge: evidence for receptorspecific serotonergic dysregulation in depressed mood. Neuropsychopharmacology 26: 358-367.

Ruiz JC, Soler MJ, Dasi C (2004). Study time effects in recognition memory. Percept Motor Skills 98: 638-642.

Schaub RT, Linden M, Copeland JRM (2003). A comparison of GMS-A/AGECAT, DSM-III-R for dementia and depression, including subthreshold depression (SD): results from the Berlin Aging Study (BASE). Int J Geriatr Psychiatry 18: 109-117.

Schmitt JAJ, Jorissen BL, Sobczak S, van Boxtel MPJ, Hogervorst E, Deutz NEP et al. (2000). Tryptophan depletion impairs memory consolidation but improves focused attention in healthy young volunteers. J Psychopharmacol 14: 21-29.

Schmitt JAJ, Kruizinga MJ, Riedel WJ (2001). Non-serotonergic pharmacological profiles and associated cognitive effects of serotonin reuptake inhibitors. J Psychopharmacol 15: 173-179.

Siegfried K, O'Connolly M (1986). Cognitive and psychomotor effects of different antidepressants in the treatment of old age depression. J Psychopharmacol 16: 207-214.

Snodgrass JG, Corwin J (1988). Pragmatics of measuring recognition memory: applications to dementia and amnesia. $J$ Exp Psychol Gen 117: 34-50.

Sohal VS, Hasselmo ME (1998). GABA-b modulation improves sequence disambiguation in computational models of hippocampal region CA3. Hippocampus 8: 171-193.

Torres GE, Arfken CL, Andrade R (1996). 5-Hydroxytryptamine4 receptors reduce afterhyperpolarization in hippocampus by 
inhibiting calcium-induced calcium release. Mol Pharmacol 50: 1316-1322.

Tóth K, Borhegyi Z, Freund TF (1993). Postsynaptic targets of GABAergic hippocampal neurons in the medial septum-diagonal band of Broca complex. J Neurosci 13: 3712-3724.

Tóth K, Freund TF, Miles R (1997). Disinhibition of rat hippocampal pyramidal cells by GABAergic afferents from the septum. J Physiol 500: 463-474.

Witter MP, Wouterlood FG, Naber PA, Van Haeften T (2000). Anatomical organization of the parahippocampal-hippocampal network. Ann NY Acad Sci 911: 1-24.

Yatham LN, Steiner M (1993). Neuroendocrine probes of serotonergic function: a critical review. Life Sci 53: 447-463.

\section{APPENDIX}

Integrate-and-fire MacGregor model neurons were used for the model. In running the simulations, the discrete-time approximation formulas given by MacGregor and Oliver (1974) were used. The model was constructed using the Nutshell simulator, developed in our group. It can be downloaded without cost at www.neuromod.org/nutshell.

MacGregor and Oliver (1974) derived their model neuron from the Hodgkin-Huxley formulas (Hodgkin and Huxley, 1952) to account for firing characteristics in single neurons, while being computationally inexpensive enough for use in large-scale networks. These model neurons show spiking, adaptation, and threshold accommodation (the latter was not implemented in the present simulations). They are updated in discrete time steps, which in our simulations lasted $2 \mathrm{~ms}$.

The model neuron emits a spike every time the membrane potential $E$ crosses the threshold $\theta$ :

$$
E>\theta \Rightarrow S=1
$$

In this equation, $S$ is a dichotomous variable that is equal to 1 if the node emits a spike, and equals 0 otherwise. The membrane potential, $E$, is dependent on the sodium, potassium, and chloride currents over the membrane, as described in the following differential equation:

$$
\begin{aligned}
\frac{\mathrm{d} E}{\mathrm{~d} t}= & -\delta E-g_{\mathrm{k}}\left(E-E_{\mathrm{k}}\right)-g_{\mathrm{ex}}\left(E-E_{\mathrm{ex}}\right) \\
& -g_{\mathrm{i}}\left(E-E_{\mathrm{i}}\right)-S E
\end{aligned}
$$

Here, $-\delta E$ is the leak current, $g_{\text {ex }}$ the excitatory conductance, $E_{\text {ex }}$ the sodium reversal potential, $g_{i}$ the inhibitory conductance, and $E_{\mathrm{i}}$ the chloride reversal potential. For computational purposes, both the membrane potential and the reversal potentials were mapped onto the interval $[-1,7]$ via a simple linear transformation (MacGregor and Oliver, 1974). Resting potential is equated to $0(-75 \mathrm{mV})$, the firing threshold $\theta$ to $1(-60 \mathrm{mV})$, the sodium reversal potential to $7(+30 \mathrm{mV})$, and both the potassium and chloride reversal potentials to $-1(-90 \mathrm{mV})$. The parameter governing the leak current, $\delta$, is set to $1 / 7$. When the node emits a spike, membrane potential is reset to resting level (via the term SE).

The potassium conductance $g_{\mathrm{k}}$ models adaptation, and is determined by

$$
\frac{\mathrm{d} g_{\mathrm{k}}}{\mathrm{d} t}=-\left(g_{\mathrm{k}} / \tau\right)+b S
$$

where $S$ is the spiking variable. The time constant $\tau$ is set to $1 / 13$, the gain parameter $b$ to 0.35 . Excitatory input to the $i$ th node is a simple linear summation of weighted inputs to that node:

$$
g_{\mathrm{ex}}=\sum_{j} w_{i j} S_{j}
$$

where $w_{i j}$ is the weight from node $j$ to node $i$, and $S_{j}$ is the spiking variable of node $j$. Rise times of synaptic inputs are thus not taken into account.

Simple Hebbian learning is used, modeling LTP, with the additions of negative Hebbian learning, modeling LTD, and a bound on connection weights. Weights are changed according to

$$
\Delta w_{i j}=\mu^{+} S_{i} S_{j}-\mu^{-} S_{i}\left(1-S_{j}\right)
$$

Here, $w_{i j}$ is the weight from node $j$ to node $i$, while $S_{i}$ and $S_{j}$ are the spiking variables of the receiving and sending node, respectively. This is subject to the constraints that a weight cannot be lower than 0 or exceed a maximum $W$. The positive learning rate, $\mu^{+}$, as well as the maximum weight, $W$, are set separately for every connection (see Table 1 ). The negative learning rate $\mu^{-}$is set to $75 \%$ of the positive learning rate in all connections.

The inhibitory conductance, $g_{i}$, in a given layer, $l$, is modeled as a continuous variable reflecting firing rates of

\begin{tabular}{|c|c|c|c|c|c|c|}
\hline & EC-DG & EC-CA3 & DG-CA3 & CA3-CA3 & CA3-CAI & EC-CAI \\
\hline Density & $40 \%$ & $40 \%$ & Three from each Dg node & $75 \%$ & $75 \%$ & One per node \\
\hline Initial weight strength & 0.09 & 0.06 & । & 0.06 & 0.08 & 0.4 \\
\hline Maximum weight & 0.18 & 0.12 & । & 0.12 & 0.2 & 0.4 \\
\hline$\mu$ & $0.04 * \Psi$ & 0.02 & 0 & $0.05 * \Psi$ & $0.05 * \Psi$ & 0 \\
\hline Dampening by ACh & & & & $\mid-0.5 * \Psi$ & $1-0.5 * \Psi$ & \\
\hline
\end{tabular}
inhibitory interneurons. It is described by the following equation:

$$
g_{i}^{l}=1+i_{t}^{l}-s_{t}
$$

Table I Parameter Values for the Different Connections in the Network

EC-DG, connection from EC to DG; Density: number of postsynaptic target nodes for each presynaptic node (absolute or as percentage from the layer) targeted by any presynaptic node; Initial weight strength, weight at the start of simulation; Maximum weight: maximum attainable weight of any connection; $\mu$, learning rate; Dampening by ACh: dampening of transmission under influence of acetylcholine; Feedforward inhibition: strength of this form of inhibition ( $\lambda$ in Equation 8 ). 
where $s_{t}$ is the activity of the septal interneuron:

$$
s_{t}=0.5-0.5 \sin (t / f \pi)
$$

This is a simple sinusoid between 0 and 1 with a frequency of $f$ (set to 50 , equivalent to a $200 \mathrm{~ms} \theta$-band oscillation). The other component of Equation $6, i_{t}^{l}$, models the activity of intrinsic interneurons:

$$
i_{t}^{l}=\alpha_{i} i_{t-1}^{l}+\beta^{l}\left(A_{t-1}^{l}\right)+\sum_{p} \lambda^{l p} A_{t-1}^{p}
$$

Thus, inhibition in layer $l$ on time step $t$ is a function of the feed-forward and feedback activation of inhibitory cells by the pyramidal cells, and of inhibition on time step $t-1$. Feed-forward and feedback inhibition are linear functions of the excitatory activation in the layers connecting to layer $l$ (feed-forward), and of excitatory activation in layer $l$ itself (feedback). The activation of each layer $\left(A^{1}\right)$ is calculated by dividing the number of firing nodes in the layer by its maximum $k^{l}\left(k^{\mathrm{EC}}=12, k^{\mathrm{DG}}=10, k^{\mathrm{CA} 3}=10, k^{\mathrm{CA} 1}=12\right)$. The $\beta^{\lambda}$ parameter (strength of feedback inhibition to layer $l$ ) was equal to 0.5 for EC, CA3, and CA1, and to 2 in layer DG. The $\lambda^{\mathrm{l} \mathrm{p}}$ parameters associated with each connection (strength of feedforward inhibition from layer $p$ to layer $l$ ) are listed in Table 1. No rise time is included in the formula for inhibition, as our $2 \mathrm{~ms}$ time step made this redundant. However, the decay parameter of the current $\left(\alpha_{l}\right)$ was set by fitting a single exponential to the double exponential used by Sohal and Hasselmo (1998); $\alpha_{l}=0.76$.

In very large networks, the inhibition described above will be sufficient to constrain activity. In networks of the size used here, random fluctuations may produce large swings in activity that can be kept in check with a fast cutoff mechanism. This mechanism allows no more than a $k^{l}$ number of nodes to fire in a layer at any given time step. If more than $k^{l}$ nodes cross the firing threshold, only the $k^{l}$ nodes with the highest membrane potential are allowed to fire.

ACh levels in the model are regulated by inhibitory activity in layers CA3 and CA1. Activity of the septal cholinergic neurons, $A_{t}^{s}$, is set to $F$-inhibition (see Equation 9). Here, $F$, set to 1 in all simulations, represents excitation of the septum by sources external to the model, such as the reticular formation. Inhibition comes from the septal oscillator interneurons, $s_{t}$ (whose output is the $\theta$-frequency sinusoid given by Equation 7), and from the hippocampal afferents, $i_{t}^{s}$. A moving average of inhibition in CA1 and CA3 determines $i_{t}^{s}$ (given by Equation 10).

$$
\begin{aligned}
& A_{t}^{s}=F-s_{t}-i_{t}^{s} \text { if } F-s_{t}-i_{t}^{s} \geqslant 0 \text { else } A_{t}^{s}=0 \\
& i_{t}^{s}=\alpha^{s} i_{t-1}^{s}+\beta^{s}\left(i_{t-1}^{\mathrm{CA} 3}+i_{t-1}^{\mathrm{CA} 1}\right)
\end{aligned}
$$

The parameter $\alpha^{s}$ is set to 0.85 , and $\beta^{s}$ to 0.45 . Release of $\mathrm{ACh}$ is equal to the activity of the septal cholinergic node, $A_{t}^{s}$. This release, in turn, determines ACh modulation in the hippocampus, for which we use the symbol $\Psi$, following Hasselmo et al (1995). At each time step, the amount of ACh released is fed into a dual exponential:

$$
\Psi=\sum_{d<t} A_{d}^{s}\left(\mathrm{e}^{-\tau_{1}(t-d)}-\mathrm{e}^{-\tau_{2}(t-d)}\right)
$$

The time constants $\left(\tau_{1}, \tau_{2}\right)$ of the dual exponential were rescaled from those found by Hasselmo and Fehlau (2001), who fitted a dual exponential to experimental data on the time course of ACh modulation data $\left(\tau_{1}=0.001258\right.$, $\tau_{2}=0.00015$ ). These values correspond to a slow rise with a maximum at around $3.5 \mathrm{~s}$, and a decrease back to 0 in $10-20 \mathrm{~s}$.

As the effects of acetylcholine have been discussed in the main text, only their implementation will be listed here.

(1) For preferential dampening of transmission over Schaffer collaterals to CA3 and CA1, transmission in these two tracts ( $g_{\text {ex }}$ in Equation 4) is multiplied by a factor $1-0.6^{*} \Psi$.

(2) For enhancement of LTP at CA3 recurrent collateral synapses and at CA1 Schaffer collateral synapses, the learning rate ( $\mu$ in Equation 5) is multiplied by $\Psi$ in these connections.

(3) Reduction of firing adaptation of DG, CA3, and CA1 excitatory cells is effectuated by multiplication of the adaptation constant ( $b$ in Equation 3) with a factor $1-\Psi$.

(4) Suppression of inhibition in all model layers is achieved multiplying the feedback inhibition constant $(\alpha$ in Equation 8 ) by a factor $1-0.5^{*} \Psi$.

(5) A mild depolarization of DG, CA3, and CA1 principle cells is implemented adding a constant factor, $0.12 * \Psi$, to the input of cells in these layers $\left(g_{\mathrm{ex}}\right.$ in Equation 4$)$.

Supplementary Information accompanies the paper on the Neuropsychopharmacology website (http://www.nature.com/npp) 